\title{
A generation effect can be found during naturalistic learning
}

\author{
PATRICIA A. DEWINSTANLEY \\ Oberlin College, Oberlin, Ohio
}

\begin{abstract}
Recently, Carroll and Nelson (1993) presented research suggesting that general-information questions might represent a boundary condition for the generation effect. The present research focused on whether the generation effect did, in fact, generalize to such questions. In Experiment 1, when subjects read or generated the answers to general-information questions, a generation advantage was demonstrated on a 47-h delayed cued-recall test. However, when the Carroll and Nelson procedure was mimicked by requiring subjects to make an initial attempt to answer the questions, the generation advantage was reduced such that it was no longer statistically significant. In Experiments 2 and 3, the findings of the first experiment generalized to a free-recall test. Thus, general-information questions do not represent a boundary condition for the generation effect.
\end{abstract}

The generation effect occurs when an individual better remembers information that he or she produces than information provided by an external source (Jacoby, 1978; Slamecka \& Graf, 1978). The generation effect has proven to be remarkably robust across a number of different paradigms and types of materials (see, e.g., Begg \& Snider's, 1987 , review of the empirical findings). Recently, however, the generality of the effect was challenged. Specifically, Carroll and Nelson (1993) cast doubt on one's ability to extend the laboratory findings to more naturalistic learning situations.

Carroll and Nelson (1993) presented subjects with general-information questions (e.g., Which planet was the last to be discovered?). A subject's task was to answer each question out loud. If the subject knew the answer, the question was discarded and another question was shown. If the subject did not know the answer, one of two procedures was followed. In the read condition, the subject was shown the question again with the answer written on a card. The subject had $15 \mathrm{sec}$ to study the question and the corresponding answer. In the generate condition, the subject was shown the question and clues to the answer. Either the clues were the number of letters in the word followed by the first letter, then the second letter, and so on until the subject correctly generated the answer (Experiment 1), or the clue was an anagram of the answer (Experiments 2-7). The recall test occurred 1 week after study (Experiments $1-4,6$, and 7) or after a 10-min distractor task (Experiment 5). Furthermore, the generation task was made either easier or

This research was supported by a grant from the Keck Foundation. I would like to thank William Friedman, Marie Carroll, Michael Pressley, Norman Slamecka, John Gardiner, Henry Roediger, and anonymous reviewers for their thoughtful comments on a previous draft. I also thank Amy Hughes for her assistance in running subjects and Gudrun Jonsdottir for scoring subjects' recall. Correspondence concerning this article should be addressed to P. A. deWinstanley, Department of Psychology, Oberlin College, Oberlin, $\mathrm{OH}$ 44074-1086 (e-mail: patty@occs.cs.oberlin. edu). more difficult by the provision of more or fewer of the letters in the anagram and the selection of easier or more difficult questions. None of these manipulations made a difference in Carroll and Nelson's studies; read and generate performances were equivalent across all but one experiment. In the experiment that revealed a generation advantage, subjects studied the answers as single-item stimuli-that is, without the questions' serving as a context.

Ironically, in the same journal issue as the Carroll and Nelson study, Peynircioglu and Mungan (1993) reported a generation effect for answers to trivia questions about sports and music. Why does one set of researchers find a generation effect and another set fail to find the effect with seemingly similar materials? The present paper presents research suggesting that Carroll and Nelson's failure to find a generation effect with naturalistic materials was due to a procedural anomaly (anomalous with respect to the standard generation-effect paradigm). The anomaly was the presentation of questions to subjects before both the read and the generate tasks, thus resulting in an attempt to generate the answers in both conditions. To their credit, Carroll and Nelson wished to extend the generation effect to more naturalistic learning situations by demonstrating that subjects did not, a priori, know the answers to the questions. However, in ensuring no knowledge of the answers, they introduced a procedural change that may itself be responsible for the lack of an effect. Indeed, previous research had demonstrated that the process of trying to answer the question is the important determinant of memory performance. In fact, a generation advantage was found after an attempt to generate an answer regardless of whether or not the subject produced the answer (Begg, Vinski, Frankovich, \& Holgate, 1991; Pressley, McDaniel, Turnure, Wood, \& Ahmad, 1987; Slamecka \& Fevreiski, 1983). Begg and his colleagues typically required a subject-in the generate conditionto attempt to generate a solution, and if the subject failed they provided the subject with the intact answer. Thus, the attempt to answer is considered the generate task. Indeed, 
Begg et al. (1991, Note 1) found that feedback after the generation attempt did not influence memory performance.

It may be that Carroll and Nelson failed to find a generation advantage because subjects for both the read and the generate conditions attempted to answer the generalinformation questions. Support for this interpretation comes from the fact that in the experiment, in which they did find a generation advantage, the read subjects studied an intact word and the generate subjects attempted to solve an anagram-no initial attempt at a solution occurred in the read condition.

In the present paper, three experiments testing the interpretation presented above are reported. The purpose of Experiment 1 was to demonstrate that a generation effect could be found with general-information questions and to demonstrate that when subjects in both the read and generate conditions were provided with an initial attempt to answer the general-information questions, the generation advantage would be eliminated. Experiments 2 and 3 demonstrated that the results of Experiment 1 generalized to a free-recall test.

\section{EXPERIMENT 1}

The procedures and stimuli in Experiment 1 mimicked the significant characteristics of Carroll and Nelson's (1993) research (i.e., the general-information questions were from the same norms and difficulty range used by Carroll and Nelson in their Experiments 2 and 3, and the read and generate tasks were blocked on the study list), with one critical exception: For half of the subjects, the read task was not preceded by an initial attempt to answer the question.

\footnotetext{
Method

Subjects and Design. The subjects were 35 Oberlin College undergraduates enrolled in an introductory-level cognitive psychology course Participation was voluntary. The encoding task was manipulated within subjects. In the read condition, the question and answer were presented on the same page in a study booklet. In the generate condition, the question and a fragment of the answer were presented on one page. The fragments were created by replacing the vowels with dashes; if the answer began with a vowel, the vowel was not replaced. The fragments were included because, without these clues, the a priori probability of the subjects' being able to answer the questions was very low. The fragments, which helped subjects to answer the questions, are typical of generationeffect experiments.

For half of the subjects ( $N=17$ ), the read and generate tasks were preceded by an initial attempt to answer the question. For these subjects. the generate and read pages were preceded by a page with the trivia question alone (i.e., without the answer or the fragment).

Materials. The stimuli were 24 questions and answers selected from the Nelson and Narens (1980) norms. Based on the information provided in the norms, the mean probability of correctly answering the questions was calculated as .25 (the range was .30 to .22 ). The study list began with the question that had the highest normative probability of being correctly answered, with each subsequent question decreasing in its normative probability. The order of questions was held constant for all subjects. The read and generate tasks were presented in alternating blocks of six items each, for a total of four blocks. To control for specific-item and order effects, one half of the study lists began with a read block and the other half of the study lists began with a generate block. Thus, every question was assigned to both the read and generate conditions equally often, across subjects. The stimuli were presented in study booklets. In half of the
}

booklets, the read and generate pages were preceded by a page with the trivia question. For example, one page would have the question "What is the monetary unit of India?" followed by either a read or a generate page. The corresponding read and generate pages had "Rupee: What is the monetary unit of India?" and "What is the monetary unit of India? $\mathrm{R}_{-} \mathrm{p}_{-}$,", respectively. In the other half of the booklets, the read and generate pages were repeated twice. Thus, all booklets had the same number of pages.

Procedure. The subjects were tested in one large group. They were told that they were participating in an experiment designed to test their memory. They were further instructed that they would be seeing generalinformation questions and that for some of the questions the answers would be provided. When the answers were not provided, the subjects were to try to generate the answers from fragments of the answer. Examples of each type of stimulus were given. In both cases, the subjects were asked to write only the answers on a page in their study booklet. They were instructed to turn the pages in their study booklets every $5 \mathrm{sec}$. Thus, every question was presented for a total of $10 \mathrm{sec}-5 \mathrm{sec}$ for either the initial attempt to answer the question or for the first instance of the generate or read task and $5 \mathrm{sec}$ for the final instance of the generate or read task. After all stimuli had been presented, the subjects engaged in a 2 -min verbal distractor task, followed by a 2 -min free-recall test of the answers. The free-recall test was given so that subjects would assume that they had completed the experiment at the conclusion of the free-recall test. Exactly $47 \mathrm{~h}$ later, a surprise cued-recall test was administered to the entire group. The cued-recall test consisted of the original questions, which were presented in the order in which they had been at study, followed by a blank space in which the subjects were instructed to write the answer.

\section{Results and Discussion}

The mean rate of failure to provide an answer to the questions at study was $8 \%$. The normative rate of failure to answer these questions, without the fragments, was $70 \%$ to $78 \%$ (Nelson \& Narens, 1980). Thus, the presence of the fragment at study greatly facilitated answering the question. For all of the experiments to follow, the analyses conditionalized upon correctly answering the questions did not differ from the analyses that were unconditional with respect to answering the questions; thus, only the unconditional analyses are presented. A two-way mixed-subjects analysis of variance was used to analyze the results for both the initial free-recall test and the delayed cued-recall test.

For the free-recall test, the mean percentage of items recalled in the generate condition $(M=38 \%)$ did not differ reliably from that in the read condition $[M=34 \% ; F(1,33)=$ $\left.1.73, M S_{\mathrm{e}}=205.47, p>.15\right]$. Furthermore, performance on the read and generate tasks did not interact with whether or not subjects were provided with an initial opportunity to attempt to answer the questions $\left[F(1,33)=0.27, M S_{\mathrm{e}}=\right.$ $205.47, p>.60]$. However, for the cued-recall test, the generate items $(M=74 \%)$ were recalled significantly more often than the read items $[M=67 \% ; F(1,33)=8.29$, $\left.M S_{\mathrm{e}}=87.54, p<.01\right]$. In addition, performance on the read and generate items interacted with whether or not subjects were given an initial opportunity to attempt to answer the trivia questions $\left[F(1,33)=4.05, M S_{\mathrm{e}}=87.54, p<.05\right]$. When subjects were not provided with this opportunity to answer the questions, performance was significantly higher for the generate items $(M=71 \%)$ than for the read items $[M=60 \% ; t(18)=3.44, p<.005]$. However, when subjects were provided with an initial opportunity to attempt to answer the trivia questions, performance on the read $(M=75 \%)$ and generate $(M=77 \%)$ items failed to dif- 
fer significantly $[t(18)=0.62, p>.50]$. The generation advantage found on the delayed cued-recall test is impressive in light of the failure to find an effect on the initial freerecall test. Thus, participation in the immediate free-recall test 2 days before the delayed cued-recall test was unlikely to have resulted in the cued-recall generation advantage.

With the same set of materials and procedures as those used by Carroll and Nelson, the present experiment demonstrated that a generation effect could be obtained with general-information questions, and that providing subjects with the general-information question before the read and generate tasks significantly reduced the generation advantage. Taken together, these results indicate that generalinformation questions do not represent a boundary condition of the generation effect. Moreover, the results suggest that earlier failures to find a generation advantage with this type of material were most probably due to the procedural anomaly of allowing subjects in the read condition to attempt an answer.

One interesting aspect of the first experiment that remains to be examined is the lack of a generation effect on the free-recall test. Two recent lines of research may be informative regarding this lack of a free-recall generation advantage. Nairne, Riegler, and Serra (1991; see also, Serra \& Nairne, 1993) assert that the requirement to generate interferes with the learning of serial-order information. Serial-order information is one component of good freerecall performance. It may be that difficult generation tasks interrupt the learning of serial-order information more than do easier tasks and that the lack of a generation advantage in the current study was due to the difficulty of the questions. Or perhaps the difficult questions induced subjects to engage in more test-appropriate processing of the read items. Begg et al. (1991) found that when read items were processed in a meaningful way, the generation effect was eliminated.

Admittedly, both of these explanations of the current results are post hoc, and distinguishing between these explanations is beyond the focus of the present research. The questions more consistent with the focus are whether or not a free-recall generation advantage can be found for answers to general-information questions and, if such an effect is obtained, will allowing an initial attempt to answer the question also eliminate the advantage on a freerecall test? The last two experiments were designed to answer these questions.

\section{EXPERIMENT 2}

If the lack of a free-recall generation effect was due to the difficulty of the questions, then selecting easier questions should result in a generation advantage. Because the purpose of the present experiment was to discover if a generation advantage could be found for general-information questions on a free-recall test, all subjects were assigned to the one condition that showed a generation effect in Experiment 1 , namely, the condition in which subjects did not attempt to answer the trivia question before doing the generate and read tasks. A cued-recall test was not used in this experiment because the questions used were relatively easy to answer and therefore would probably produce a ceiling effect.

\section{Method}

The method used in Experiment 2 was identical to that used in Experiment 1 except as noted below.

Subjects and Design. The subjects were 29 undergraduate students enrolled in an introductory psychology course at Oberlin College. Participation partially fulfilled a course requirement. The between-subjects manipulation of whether or not subjects were allowed an initial attempt to answer the trivia questions was not implemented in this experiment.

Materials and Apparatus. The stimuli were 24 general-information questions selected from the Nelson and Narens (1980) norms. The mean normative probability of correctly answering the questions was calculated as .87; the range was .78-.97. In addition, because the questions were relatively easy, the provision of fragments in the generate condition was unnecessary. The stimuli were presented on DOS-based computers. Response booklets were constructed with 24 pages so that the subjects could write one answer per page.

Procedure. The subjects were tested in groups of 2-5, sitting individually at a computer terminal. The stimuli were presented for $10 \mathrm{sec}$, followed by a blank screen for $1 \mathrm{sec}$ and then the next stimulus in the series.

\section{Results and Discussion}

The mean rate of failure to provide an answer to the questions at study was $14 \%$. A two-tailed dependentgroups $t$ test was used to analyze the results. Significantly more generate items $(M=44.03 \%)$ were recalled than read items $[M=36.66 \% ; t(28)=2.10, p<.05]$. Thus, it seems that a generation advantage can be found if the general-information questions are relatively easy. Experiment 3 was designed to determine whether providing an initial attempt to answer the general-information questions before both the read and generate tasks would eliminate the generation advantage found in Experiment 2.

\section{EXPERIMENT 3}

The prediction for Experiment 3 was that the generation advantage seen in Experiment 2 would be eliminated by the inclusion of initial attempts to answer the questions.

\section{Method}

Except where indicated below, all methods used in Experiment 3 duplicated those used in Experiment 2.

The subjects were 21 Oberlin college undergraduates enrolled in an introductory psychology course. Participation partially fulfilled a course requirement. The question appeared on the computer screen for $5 \mathrm{sec}$. followed by the generate or read stimulus for another $5 \mathrm{sec}$. A blank screen was presented for $1 \mathrm{sec}$ between trials.

\section{Results and Discussion}

The mean rate of failing to provide an answer to the general-information questions at study was $15 \%$. Recall in the read condition $(M=36.10 \%)$ did not differ significantly from recall in the generate condition $[M=31.52 \%$; $t(20)=0.89, p>.30]$.

The last two experiments again demonstrated that general-information questions do not represent a boundary condition for the generation effect. A generation advantage for the answers to general-information questions can be found on both cued-recall and free-recall tests. Further- 
more, in both cases, the advantage is eliminated by an initial attempt at answering the questions.

\section{GENERAL DISCUSSION}

The results of the present set of experiments fit in nicely with the body of research on the generation effect that suggests that the effect can be generalized to real-world settings. In fact, a large body of research exists on the generation effect with materials very much like those found in an educational setting (see, e.g., Doctorow, Wittrock, \& Marks, 1978; Gardiner \& Rowley, 1984; Graf, 1980; Pressley et al., 1987; Wittrock, 1990 Wittrock \& Carter, 1975). I believe that the present three experiments illuminate the reason why Carroll and Nelson (1993) failed to obtain a generation effect with naturalistic materials when, as mentioned above, a vast body of research suggests that the generation effect can be extended to naturalistic materials. In conclusion, the generation effect is not limited to traditional laboratory situations; it has been repeatedly extended to materials and situations like those encountered in the real world. As such, the generation effect is an important memory phenomenon that warrants further attention.

\section{REFERENCES}

BEGG, I., \& SNider, A. (1987). The generation effect: Evidence for generalized inhibition. Journal of Experimental Psychology, 13, 553-563

BegG, 1., Vinski, L., Frankovich, L., \& Holgate, B. (1991). Generating makes words memorable, but so does effective reading. Memory \& Cognition, 19, 487-497.

Carroll, M., \& Nelson, T. O. (1993). Failure to obtain a generation effect during naturalistic learning. Memory \& Cognition, 21, 361-366.

Doctorow, M., Wittrock, M. C., \& Marks, C. (1978). Generative processes in reading comprehension. Journal of Educational Psychology, 70, 109-118.

Gardiner, J. M., \& Rowley, J. M. C. (1984). A generation effect with numbers rather than words. Memory \& Cognition, 12, 443-445.
GraF, P. (1980). Two consequences of generating: Increased inter- and intraword organization of sentences. Journal of Verbal Learning \& Verbal Behavior, 19, 316-327.

JACOBY, L. (1978). On interpreting the effects of repetition: Solving a problem versus remembering a solution. Journal of Verbal Learning \& Verbal Behavior, 17, 649-667.

Nairne, J. S., Riegler, G. J., \& Serra, M. (1991). Dissociative effects of generation on item and order information. Journal of Experimental Psychology: Learning, Memory, \& Cognition, 17, 702-709.

NELSON, T. O., \& NARENS, L. (1980). Norms of 300 general-information questions: Accuracy of recall, latency of recall, and feeling-of-knowing ratings. Journal of Verbal Learning \& Verbal Behavior, 22, 338-368.

Peynirchioglu, Z. F., \& Mungan, E. (1993). Familiarity, relative distinctiveness, and the generation effect. Memory \& Cognition, 21, 367-374

Pressley, M., McDaniel, M. A., Turnure, J. E., Wood, E., \& AhMAD, M. (1987). Generation and precision of elaboration: Effects on intentional and incidental learning. Journal of Experimental Psychology: Learning, Memory, \& Cognition, 13, 291-300.

SerRA, M., \& NaIRNE, J. S. (1993). Design controversies and the generation effect: Support for an item-order hypotheses. Memory \& Cognition, 21, 34-40.

Slamecka, N. J., \& Fevreiski, J. (1983). The generation effect when generation fails. Journal of Verbal Learning \& Verbal Behavior, 22, 153-163.

Slamecka, N. J., \& Graf, P. (1978). The generation effect: Delineation of a phenomenon. Journal of Experimental Psychology: Human Learning \& Memory, 4, 592-604.

WiTTROCK, M. C. (1990). Generative processes of comprehension. Educational Psychologist, 24, 345-376.

WitTrock, M. C., \& CarTeR, J. F. (1975). Generative processing of hierarchically organized words. American Journal of Psychology, 88, 489-501.

(Manuscript received September 16, 1994; revision accepted for publication April 12, 1995.)

\section{Cognitive Science Program National Institute of Mental Health}

The Cognitive Science Program of the National Institute of Mental Health (NIMH) invites grant applications for basic research in such areas as perception, action, attention, memory, learning, knowledge, reasoning, and language. Methods may be drawn from psychology, artificial intelligence, linguistics, philosophy, and neuroscience. Research may be conducted with humans or other animals, at any stage in the lifespan. Purely computational work is supported, but it should make substantive contact with behavioral or physiological evidence.

Funding mechanisms include grants for research projects (of various sizes and durations), institutional training grants, pre- and postdoctoral fellowships, career scientist awards, and conference grants. Grants are awarded primarily, but not solely, to institutions in the U.S. Individual inyestigators need not be U.S. citizens; however, recipients of pre-/postdoctoral fellowships and career scientist awards must be U.S. nationals or permanent residents.

General information and application kits for NIMH grants are available at most U.S. institutions' research offices. (Information is also available electronically via gopher.nimh.nih.gov.) Further questions can be directed to

Howard S. Kurtzman, Ph.D.

Chief, Cognitive Science Program

National Institute of Mental Health

5600 Fishers Lane, Rm. $11 \mathrm{C}-16$

Rockville, MD 20857 